\title{
The range effect as a function of stimulus set, presence of a standard, and modulus
}

\author{
KATHLEEN H. KOWAL \\ University of North Carolina, Wilmington, North Carolina
}

\begin{abstract}
The intramodal range effect (an inverse relationship between stimulus range and exponent in Stevens's power law) has been well documented, but its conditions have not been tested. Both the estimates of stimulus magnitudes and their exponents are affected by context, stimulus location, and different standards and moduli, but how these variables might interact with the variable of stimulus range has not been studied. In the present research, exponents were derived from magnitude estimates of line length for each of three different stimulus ranges at two different locations on the scale of length, with or without a modulus. Moduli of 50 and 500 permitted an analysis of the effect of response magnitude on the range effect. Because different ranges had stimulus values in common, the effect of range and location on exponents from those common values could be determined. Exponents decreased as stimulus range increased, but only in the free-modulus condition. For that condition, exponents derived from magnitude estimates of only the common stimuli also showed the range effect and response magnitude did not influence the range effect. Exponents were higher for stimulus ranges at the lower location, but location does not appear to contribute to the range effect. Although the range effect is not explained, the conditions under which it holds and some factors that may influence it are considered.
\end{abstract}

The intramodal range effect is the decrease in the exponent of S.S. Stevens' power law for a given target modality as the log stimulus range increases. The effect has been well documented (Ahlström \& Baird, 1989; Engen, 1956; Engen \& Levy, 1958; Foley, Cross, Foley, \& Reeder, 1983; Jones \& Woskow, 1966; Kunnapas, 1960; R. Teghtsoonian, 1973; R. Teghtsoonian \& M. Teghtsoonian, 1978) for several stimulus continua, but not explained satisfactorily. The role of one factor-location of the stimulus range along the stimulus continuum-in determining the range effect has only recently received attention (Ahlström \& Baird, 1989; Fagot \& Pokorny, 1989; Foley et al., 1983; Foley, Cross, \& O'Reilly, 1990; Marks, 1988). Response magnitude, a second factor that might contribute to the range effect, has not been studied systematically. In the present study, the effects of both stimulus location and response magnitude on the exponent for magnitude estimates of length were explored in an attempt to understand the range effect. Response range, a third factor that is often considered in explanations of the range effect (Poulton, 1989), is not directly manipulated. However, because response range may vary with response magnitude, its role in the range effect is considered.

\section{Stimulus Location, Response Magnitude, and the Range Effect}

When the stimulus range is changed, so are the magnitudes of the particular stimuli being judged. Thus, to

I would like to thank Martha Teghtsoonian and Julian Hochberg for their suggestions and comments on the manuscript, and Judy Jones Clark for her help in conducting these experiments. Address correspondence to K. H. Kowal, Department of Psychology, University of North Carolina, Wilmington, NC 28403-3297. increase a particular stimulus range, one could alter the identity of the original stimulus range only partially by adding stimuli at the top and/or bottom of the range, or completely by moving the range to a different location that includes a different set of stimuli. Because stimulus range and location are often changed together, effects on the exponent might be due to either.

Varying the stimulus range by changing the location of the stimuli or the context of some core of common stimuli may modify the way the sensory magnitudes of the stimuli are perceived or judged. In studies in which stimulus location has been manipulated, results have been inconclusive. Exponents were greater at the lower than at the upper end of the continuum for judgments of sound intensity (Ahlström \& Baird, 1989; Foley et al., 1990) and heaviness (Fagot \& Pokorny, 1989). However, Marks (1988) reported no difference in exponents, whereas Fagot and Pokorny reported smaller exponents at the lower end than at the upper end of the sound intensity continuum. For judgments of length, the continuum of interest in the present study, exponents are typically near 1 (Gescheider, 1985). For different ranges and locations, exponents have been found to vary from .79 to 1.43 (Foley et al., 1983), with no pattern emerging for different locations (M. Teghtsoonian, personal communication, August, 1992).

Location of the stimulus range may not influence the exponent directly; rather, changes in location may induce subjects to use different numbers in responding to different ranges (with a free-modulus method). For example, ranges having larger stimulus values may encourage the use of larger numbers, and ranges having smaller stimulus values may encourage the use of smaller numbers. If subjects use small and large numbers differently, what 
appear to be range effects may only be artifacts of the section of the number continuum selected by subjects for each range. A design that controls for this possible artifact would ensure that the subject judges the same range at different locations with numbers of similar magnitude.

\section{The Effect of a Standard and a Modulus on the Exponent}

A response magnitude hypothesis can be tested by measuring the exponent for the same stimulus ranges while manipulating response magnitude by the use of a standard and a modulus. In studies that have examined the effect of a modulus and a standard on the exponent, results have been inconclusive. Some have shown no effect (Fagot $\&$ Pokorny, 1989, for loudness; Wiest \& Bell, 1985), whereas others have shown that exponents are greater when a standard and a modulus are present (Fagot \& Pokorny, 1989, for heaviness; MacMillan, Moschetto, Bialostozky, \& Engel, 1974; J. C. Stevens \& Tulving, 1957; S. S. Stevens \& Galanter, 1957). How the exponent is affected appears to vary with the location of the standard (Poulton, 1968) and the size of the modulus (Engen \& Ross, 1966; Wong, 1963).

In 1971, S. S. Stevens concluded that it is better not to designate a standard and a modulus, and since that time the majority of magnitude estimation studies have followed his recommendation. When a standard and a modulus are included in studies of the range effect, the occurrence of the effect depends on the standard's magnitude. For example, Ahlström and Baird (1989) varied range and location but used the same standard throughout, so that it was (1) at the top of a small-range, low-location set of stimuli, (2) at the bottom of a small-range, high-location set, and (3) in the middle of a full-range set. Exponents for the full range were smaller than those for low-location small ranges but greater than those for high-location small ranges. These mixed findings might have been due to the location of the ranges or to the relative value of the standard.

A standard with its modulus may serve as a scale unit that remains stable, regardless of the range of stimulus values being judged. In the absence of an external scale unit, which is the case in free-modulus tasks, the scale unit used by subjects is normally generated from, and may vary with, the particular range of stimuli presented. Such a floating scale unit might change as the stimulus range is changed by the addition or substitution of larger or smaller stimuli. Subjects' judgments of a particular stimulus, and possibly exponents as well, might depend on whether that stimulus came from a range of small stimulus values with a small floating standard or from one of larger stimulus values with a larger floating standard. An examination of the range effect in the presence and absence of standards and moduli, and with moduli of different values, would clarify the role of moduli and response magnitudes in that effect.

Also, the manipulation of response magnitude may provide a test of a constant response range explanation of the range effect. A version of this hypothesis states that exponents decrease with increases in stimulus range because subjects use a constant response range (Poulton, 1989). When Stevens's power law perfectly describes a subject's responses, the exponent equals the ratio of the $\log$ response range to the log stimulus range. With the response range constant, the exponent would automatically decrease as stimulus range is increased. This explanation would predict a constant response range, regardless of response magnitude and stimulus range.

\section{The Present Experiment}

In the present study, the range effect was examined as a function of stimulus range, stimulus location, and response magnitude. Three ranges of stimulus lengths were presented as having either predominantly short or predominantly long lengths. To examine whether the same stimulus values embedded in different ranges show the range effect, the design made stimuli common to the ranges at each location. To manipulate response magnitude, the procedure required subjects to make magnitude estimates in either a free-modulus condition or with a modulus of 50 or 500 .

\section{METHOD}

\section{Subjects}

One hundred and forty-four undergraduate students from classes in introductory psychology at the University of North Carolina at Wilmington participated to fulfill a course requirement.

\section{Materials}

Twenty-four lengths of plastic-coated wire (20 ga) ranging from .5 to $195 \mathrm{~cm}$ served as stimuli. Lines of 7,11 , and $7.35 \mathrm{~cm}$, each drawn on a separate piece of poster paper $(56 \times 71.5 \mathrm{cn})$, served as standards for ranges lower along the stimulus continuum (Ranges $A, B$, and C, respectively). Lines of 90,75 , and $50 \mathrm{~cm}$, each drawn on a separate piece of poster paper $(71 \times 111.5 \mathrm{~cm})$, served as standards for ranges higher along the stimulus continuum (Ranges $X$, $Y$, and $Z$, respectively). Each line served as a standard for one of six ranges and its length was the geometric mean of the stimulus values within the range. Stimulus lengths for each range were 2 , $3,4,5,6,7,9,11,13,14,15 \mathrm{~cm}$ (Range A); 2, 3, 5, 7, 11, 15, $18,22,26,30,34 \mathrm{~cm}$ (Range B); .5, 1, 2, 3, 5, 7, 11, 15, 18, $22,26,30,34 \mathrm{~cm}$ (Range C); 26, 34, 51, 63, 79, 99, 103, 111, $128,145,162,179,195 \mathrm{~cm}$ (Range X); 11.5, 22, 34, 51, 63, 79, $99,111,128,145,162,179,195 \mathrm{~cm}$ (Range $Y$ ); and 2.9, 6, 11, $15,22,34,51,79,88,99,103,111,128,145,162,179,195 \mathrm{~cm}$ (Range $\mathrm{Z}$ ). The number of stimuli was greater for the larger ranges and the larger stimuli (e.g., 11 stimuli in Range $A$ and 17 in Range $Z$ ) so that the linear distances between stimuli would not be too large. The choice of stimulus lengths was quasi-random and guided by the following constraints: (1) log stimulus ranges must be equal at the low and high end of the continuum; (2) in some cases, stimulus lengths were the same as those used by others; (3) lengths could neither be too short nor too long to work with; and (4) the number of units between consecutive lengths varied so that the subjects could not determine a pattern. To permit comparisons of the same stimulus values when they were embedded within different ranges, several stimuli were common to several of the ranges.

\section{Procedure}

The subjects were randomly assigned to one of the following six stimulus range conditions-two short ranges of $.88 \mathrm{log}$ units, 
Range A (2-15 cm) at the lower and Range X $(26-195 \mathrm{~cm})$ at the higher location; two moderate ranges of 1.23 log units, Range $B$ $(2-34 \mathrm{~cm})$ at the lower and Range $Y(11.5-195 \mathrm{~cm})$ at the higher location; and two long ranges of $1.83 \mathrm{log}$ units, Range $C(.5-34 \mathrm{~cm})$ at the lower and Range $\mathrm{Z}(2.9-195 \mathrm{~cm})$ at the higher location.

Within each range condition, 8 subjects were randomly assigned to each of three modulus groups: free-modulus, modulus of 50, and modulus of 500 . For each range condition there were two orders of stimulus presentation, and within each range and modulus condition half of the subjects were assigned to each order of presentation. One order was random, with the constraints that the first and last stimuli were neither the longest nor the shortest; the other order was the reverse of the first.

The subjects, run individually, sat at a table $(60 \times 181.5 \mathrm{~cm})$ in a quiet cubicle. All judgments of length were made by the technique of magnitude estimation. In the free-modulus condition, the subjects were instructed to judge the length of each wire by assigning it a number such that the magnitude of the number indicated the length of the wire. They were told to choose any number for the first length (except zero or negative numbers) and to make judgments of subsequent lengths relative to that of the first. If, for example, they called the first length 100 and the second seemed twice as long, they were to assign it the number 200 . In the two modulus conditions, the poster board showing the standard length was hung on the wall at eye level above the desk and remained in view throughout the experiment. The subjects were instructed in the use of magnitude estimation and were told to judge each length relative to the standard length, which had been assigned the number 50 or 500 . For example, if the first length seemed twice as long as the standard, they were to assign it the number 100 or 1,000 . The rest of the procedure was the same as that for the free-modulus condition. The experimenter stretched the first wire out on the table in front of the subject. After each judgment was given, the experimenter recorded it, removed the wire, and presented the next wire. The subjects were not allowed to refer back to previous stimuli or to their judgments.

Within each range condition, the entire sequence of stimuli was presented three times, always with the same order of presentation. The one exception, Range $Z$, was presented only twice because of the larger number of stimuli within that range.

\section{RESULTS}

\section{Response Magnitude as a Function of Modulus Condition}

We must first discover whether response magnitude was manipulated successfully before asking how it influences the exponent and range effect. An analysis of variance (ANOVA; modulus $\times$ stimulus range $\times$ location) on the means of the logarithms of each subject's estimates provides this information. The geometric means of all the subjects' responses (and the means of the log response ranges) for each location, range, and modulus condition are presented in Table 1. (Geometric means show actual response magnitude; logarithms, used in the ANOVA, reduce skew and equate the variances in the different conditions. The logarithms of the geometric means equal the means of the logarithms of all the subjects' responses.) Mean $\log$ estimates increased from the free-modulus to the modulus-50 to the modulus-500 condition $[F(2,126)=$ $646.20, p<.01]$. The effect of location $[F(1,126)=$ $68.40, p<.01]$, the modulus $\times$ location interaction $[F(2,126)=77.20, p<.01]$, and tests of simple main
Table 1

Geometric Means of Subjects' Magnitude Estimates and Means of Log Response Ranges of Individual Subjects as a Function of Modulus, Stimulus Range, and Location

\begin{tabular}{lrrr}
\hline & \multicolumn{3}{c}{ Modulus Condition } \\
\cline { 2 - 4 } Range & Free & 50 & \multicolumn{1}{c}{500} \\
\hline & Lower Location & & \\
A (2-15 cm) & & & \\
Geometric Mean & 2.85 & 47.20 & 465.46 \\
Log Response Range & 1.13 & 1.04 & 1.01 \\
B (2-34 cm) & & & \\
Geometric Mean & 4.96 & 48.82 & 490.14 \\
Log Response Range & 1.42 & 1.28 & 1.29 \\
C (.5-34 cm) & & & \\
Geometric Mean & 3.63 & 44.85 & 523.60 \\
Log Response Range & 1.71 & 2.02 & 1.72 \\
& & & \\
X (26-195 cm) & & & \\
Geometric Mean & 25.43 & 47.75 & 490.17 \\
Log Response Range & .98 & .95 & .87 \\
Y (11.5-195 cm) & & & \\
Geometric Mean & 46.36 & 47.98 & 439.91 \\
Log Response Range & 1.25 & 1.26 & 1.23 \\
X (2.9-195 cm) & & & \\
Geometric Mean & 32.97 & 42.51 & 448.40 \\
Log Response Range & 1.81 & 1.84 & 1.96 \\
\hline
\end{tabular}

effects showed that estimates of stimuli at the lower end of the continuum were smaller in the free-modulus condition only. Neither the effect of range $[F(2,126)=1.70$, $p>.05]$ nor the remaining interactions between the variables was significant. Thus, the analysis confirmed the effectiveness of modulus assignment in influencing subjects' choices of response (modulus- 50 conditions had an overall mean response of 46.5 ; modulus-500, 476.3), regardless of range or location. It also showed that the subjects' responses in the free-modulus condition were sensitive to location (responses in lower locations had an overall mean of 3.8; in higher locations, 34.9). Finally, it indicated that responses in the assigned-modulus conditions were greater than those in the free-modulus condition, whose overall mean was 19.4.

\section{The Effect of Stimulus Range, Location, and} Modulus on the Exponent of Stevens's Power Law

Given that response magnitude was successfully manipulated, we can examine the effects of response magnitude on the exponents and the range effect. Exponents of the best-fitting power functions were determined from the logarithmically transformed geometric means of each subject's multiple (two or three) estimates of each stimulus by the method of least squares. The Pearson $r^{2}$ values for these functions ranged from .935 to .998 . Means of the individual exponents are plotted in Figure 1 for each stimulus range and modulus condition. Standard deviations for these exponents varied from .14 to .24 in the freemodulus condition, from .10 to .18 in the modulus- 50 condition, and from .11 to .17 in the modulus- 500 condition. Geometric means of all the subjects' magnitude estimates of length for each range, location, and modulus condi- 


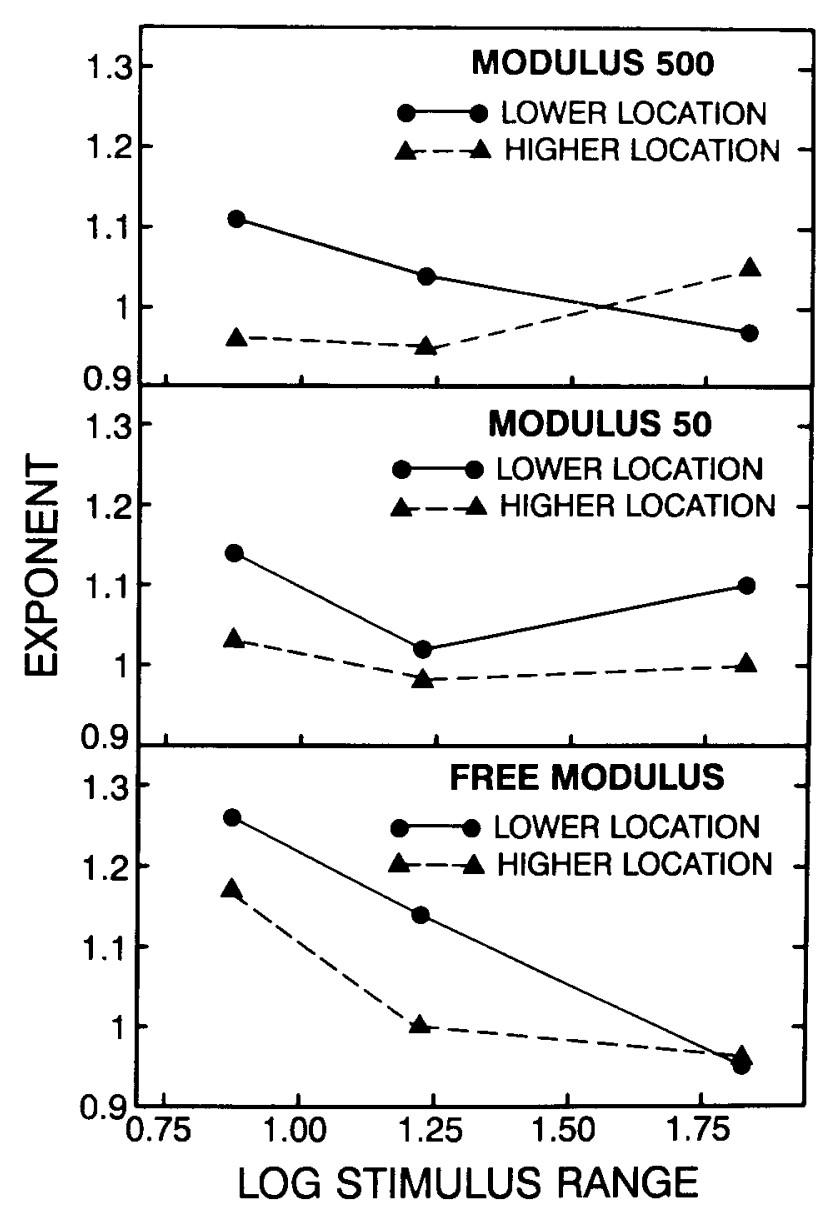

Figure 1. Means of the individual exponents derived from magnitude estimates of length as a function of log stimulus range, modulus, and location along the stimulus continuum.

tion, and the best-fitting straight lines for these functions are shown in Figures 2, 3, and 4.

An ANOVA (range $\times$ location $\times$ modulus) was performed on the individual exponents. If there is a range effect quite independent of response magnitude, then we would expect a significant effect of range in each modulus condition. Exponents decreased as stimulus range increased $[F(2,126)=6.85, p<.01]$, but not in all the modulus conditions. The significant modulus $\times$ range interaction $[F(2,126)=3.00, p<.05]$ and tests of simple main effects showed that the range effect was significant only in the free-modulus condition, with exponents for the shortest ranges being greater than for the moderate $(p<.05)$ and longest $(p<.01)$ ranges.

If response magnitude accounts for the range effect, we would expect an effect of modulus size when location and range are equated. Neither the presence of a modulus nor its value had an effect on the exponent $[F(2,126)=2.08$, $p>.05]$. Further, if the exponent varied with response magnitude, we would expect a higher exponent in the lower location for the free-modulus condition, because only there did response magnitude change with location. This was not the case. Exponents were significantly greater for stimulus ranges at the lower location, regardless of the modulus condition $[F(1,126)=7.35, p<.01]$ and there was no modulus $\times$ location interaction $[F(2,126)=$ $.11, p>.05$ ].

If the range effect is independent of location, we would expect the following in the free-modulus condition: (1) Increasing the range by adding stimuli at the top (for ranges at the lower location) would have the same effect as increasing the range by adding stimuli at the bottom (for ranges at the higher location), and (2) the range effect should be similar at both locations. In the freemodulus condition, range had a similar effect at the two locations $[F(2,126)=1.73, p>.05]$.

If the location effect (greater exponents for stimulus ranges at lower locations) were to contribute to the range effect, then we might expect a range effect when there is a location effect. This was not the case. The location effect occurred in all modulus conditions and was, in most cases, as large in the assigned modulus conditions as it was in the free-modulus condition (see Figure 1), whereas the range effect occurred only in the free-modulus condition. Although it appears from Figure 1 that the location effect varies with range and modulus conditions, the range $\times$ location $\times$ modulus interaction was not significant $[F(4,126)=1.06, p>.05]$.

If the range effect can be explained by a constant response range hypothesis, we would expect response ranges to be similar across the different stimulus ranges in the

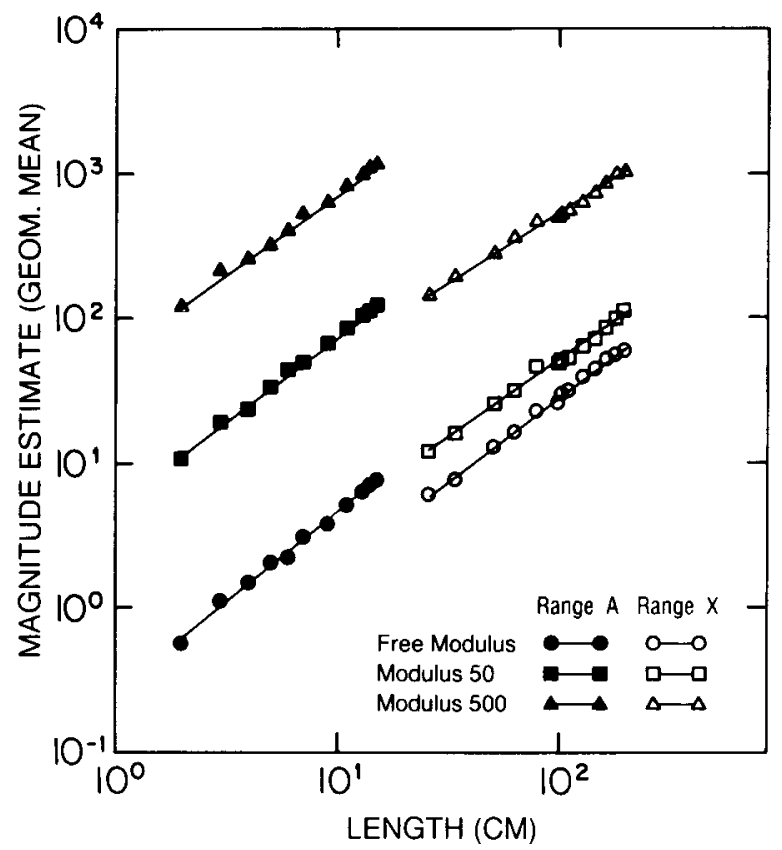

Figure 2. Geometric means of all the subjects' magnitude estimates of length (and best-fitting straight lines) for the short stimulus ranges at the lower (Range $A$ ) and higher (Range $X$ ) locations under the three modulus conditions. 


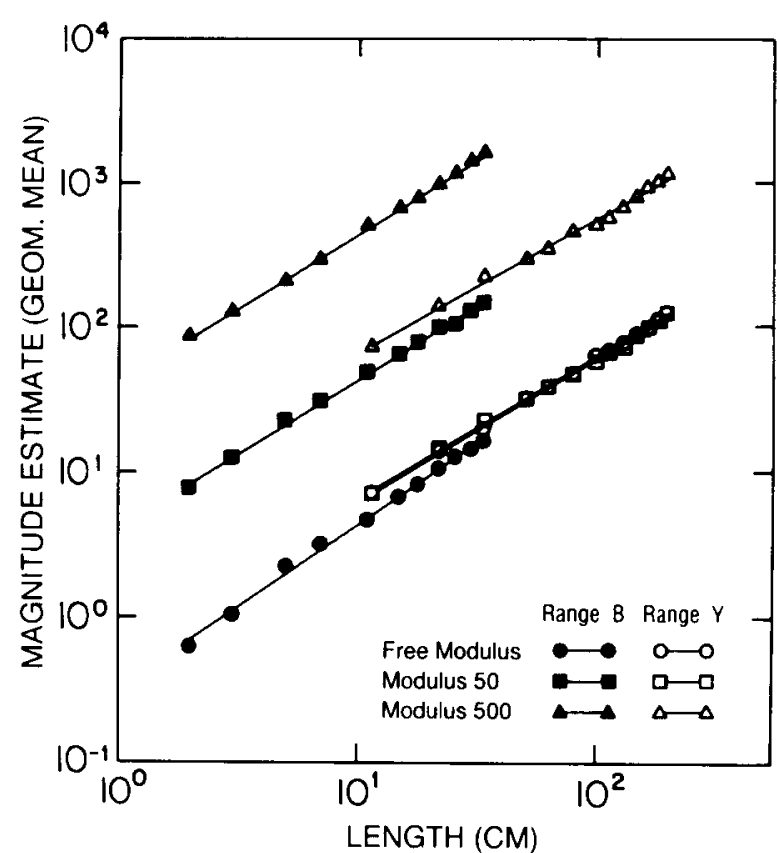

Figure 3. Geometric means of all the subjects' magnitude estimates of length (and best-fitting straight lines) for the moderate stimulus ranges at the lower (Range B) and higher (Range $Y$ ) locations under the three modulus conditions.

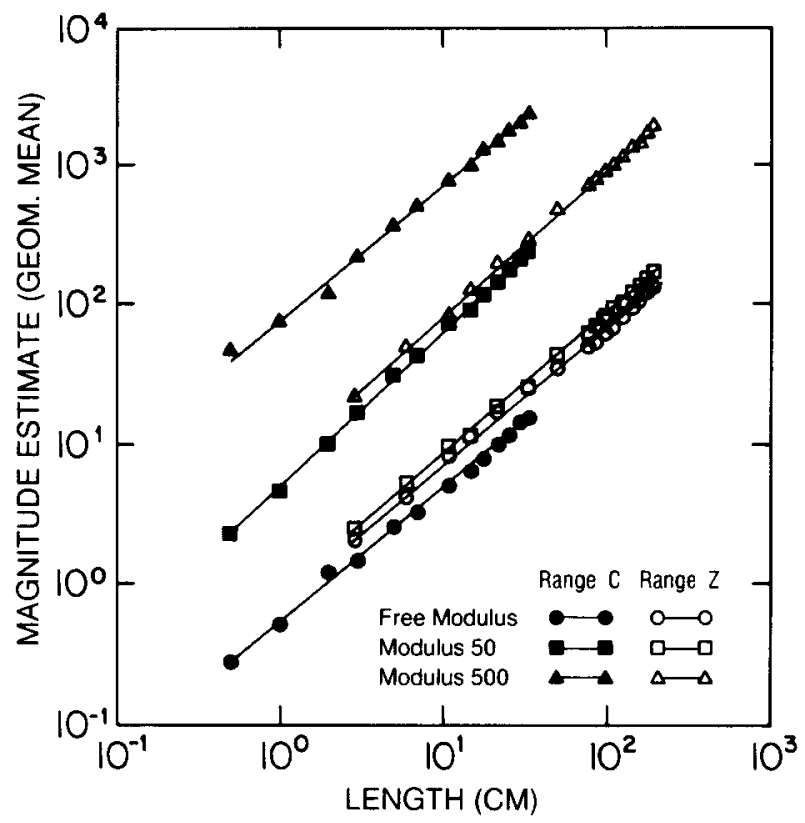

Figure 4. Geometric means of all the subjects' magnitude estimates of length (and best-fitting straight lines) for the long stimulus ranges at the lower (Range $C$ ) and higher (Range $Z$ ) locations under the three modulus conditions. free-modulus condition where there is a range effect, but to increase with increases in stimulus range in the assigned modulus conditions where there is no range effect. An ANOVA (modulus $\times$ stimulus range $\times$ location) on the log response ranges (see Table 1 for means of $\log$ response ranges) showed that log response range increased as stimulus range increased $[F(2,126)=204.85, p<$ $.01]$ and that this increase was similar for all the modulus conditions [the modulus $X$ range interaction was not significant; $F(4,126)=1.96, p>.05$ ]. Log response range did not change significantly with modulus condition $[F(2,126)=.81, p>.05]$ or location $[F(1,126)=$ $2.54, p>.05]$, and none of the remaining interactions were significant.

The range effect was observed for stimulus ranges at two locations, but only when no modulus was presented; given a modulus, the exponent appears stable across stimulus ranges. There was a location effect-greater exponents at the lower location-that was independent of range and that occurred in all the modulus conditions. Neither a response magnitude hypothesis of the range effect nor a constant response range hypothesis predicted the outcomes.

\section{The Effect of Response Magnitude on the Range Effect for Exponents of Common Stimuli}

The absence of a range effect in the assigned modulus conditions does not rule out a response magnitude explanation of the effect in the free-modulus condition. The use of a standard and modulus might have eliminated the range effect, or responses may have been so large in the assigned modulus conditions (see Table 1) that they exceeded the range of response magnitudes for which fluctuations in the exponent occur. To consider whether a response magnitude hypothesis might explain the range effect in the free-modulus condition, the only condition in which response magnitude varied with location, exponents from the free-modulus condition were analyzed further.

Exponents and intercepts were derived for each subject from estimates of the stimuli that were common to the three ranges at each location. Stimuli common to Ranges A, B, and C were 2, 3, 5, 7, 11 , and $15 \mathrm{~cm}$; stimuli common to Ranges $X, Y$, and $Z$ were $34,51,79,99$, $111,128,145,162,179$, and $195 \mathrm{~cm}$. The analysis was confined to common stimuli for two reasons: (1) to study the effect of response magnitude independent of different stimulus values, and (2) to determine whether the same stimulus values are judged differently, in that they produce different exponents when they are embedded in different stimulus ranges. Within each range condition, the subjects were divided into two equal groups at the median of the geometric mean of responses to the common stimuli. At lower locations, the below- and above-median groups had geometric mean responses of 1.69 and 3.87, respectively; at higher locations, 24.99 and 118.54 . Exponents from the below- and above-median groups are shown in Table 2. An ANOVA (response magnitude $x$ 
Table 2

Means $(M)$ and Standard Deviations $(S D)$ of Exponents of Individual Subjects Derived from Magnitude Estimates of Common Stimuli in the Free-Modulus Condition as a Function of Response Magnitude, Stimulus Range, and Location

\begin{tabular}{|c|c|c|c|c|}
\hline \multirow[b]{3}{*}{ Range } & \multicolumn{4}{|c|}{ Response Magnitude } \\
\hline & \multicolumn{2}{|c|}{ Small } & \multicolumn{2}{|c|}{ Large } \\
\hline & $M$ & $S D$ & $M$ & $S D$ \\
\hline \multicolumn{5}{|c|}{ Lower Location } \\
\hline A $(2-15 \mathrm{~cm})$ & 1.21 & .20 & 1.32 & .15 \\
\hline B $(2-34 \mathrm{~cm})$ & 1.35 & .42 & 1.02 & .05 \\
\hline$C(.5-34 \mathrm{~cm})$ & .94 & .11 & .79 & .22 \\
\hline \multicolumn{5}{|c|}{ Higher Location } \\
\hline$X(26-195 \mathrm{~cm})$ & 1.21 & .23 & 1.17 & .22 \\
\hline$Y(11.5-195 \mathrm{~cm})$ & 1.04 & .15 & .97 & .12 \\
\hline$Z(2.9-195 \mathrm{~cm})$ & .79 & .23 & 1.13 & .08 \\
\hline
\end{tabular}

range) was performed to compare below- and abovemedian exponents for each location separately. Response magnitude did not affect the exponents at either the lower $[F(1,18)=1.60, p>.05]$ or the higher $[F(1,18)=$ $1.33, p>.05]$ location; exponents decreased as stimulus range increased at both the lower $[F(2,18)=7.10, p<$ $.01]$ and the higher $[F(2,18)=4.00, p<.05]$ locations. The interaction was not significant at either the lower $[F(2,18)=2, p>.05]$ or the higher $[F(2,18)=3.33$, $p>, 05]$ location.

From this analysis, it appears that the range effect was not a function of the magnitude of the subjects' responses, because it occurred for both large- and small-number users in the free-modulus condition. Thus, the range effect may be eliminated by the presence of a modulus per se, at least when its value is 50 or 500 , rather than by the larger responses encouraged in the assigned modulus conditions. However, these results must be interpreted with caution. Responses of the subjects in the assigned modulus conditions centered around 50 or 500 (see Table 1), while even the large-number users in the free-modulus conditions did not use responses of those magnitudes. For the one group that did (the large-number users at the higher location), exponents did not always decrease as range increased (see Table 2).

\section{DISCUSSION}

Exponents of Stevens's power law derived from magnitude estimates of length were found to range from .95 to 1.26 and to fall within the range of values reported by others. In both the present and previous studies (Ahlström \& Baird, 1989; Bjorkman \& Strangert, 1960; Engen, 1956; Engen \& Levy, 1958; Foley et al., 1983; Jones \& Woskow, 1966; Kunnapas, 1960; R. Teghtsoonian, 1973), the exponent of Stevens's power law decreased with increases in intramodal stimulus range. However, such a range effect occurred here only when there was no assigned standard and modulus. In the free-modulus conditions, the inverse relation between stimulus range and exponent was demonstrated both when the exponent was based on responses to all stimulus values within each of the three ranges and when it was based only on responses to stimulus values common to all ranges. Thus, judgments of the same stimuli, as measured by exponents, may vary when the stimuli are embedded within different ranges.

The exponent varies with the location of equal-sized stimulus ranges along the stimulus continuum. Consistent with some reports (Ahlström \& Baird, 1989; Foley et al., 1990 , Experiment 2), exponents are greater for stimulus ranges located lower on the stimulus continuun than for the same-sized ranges located higher on the stimulus continuum. Although the location effect may be due to differences in stimulus spacing for the same ranges at the two locations, it is not likely. At the lower locations, logarithmic spacings between stimulus values were greater than for the same ranges at the higher locations. (The mean log spacings were $.20, .28$, and .35 for Ranges $\mathrm{A}$, $B$, and $C$ at the lower and $.17, .24$, and .28 for Ranges $\mathrm{X}, \mathrm{Y}$, and $\mathrm{Z}$ at the higher location). According to Poulton (1989, p. 94), the exponent is higher for smaller than for larger stimulus spacing, and thus exponents should have been larger for the smaller stimulus spacings at the higher location-the opposite of our results. The exponent might also vary with the geometric mean of the stimuli (Foley et al., 1983), but that would not explain the location effect in our data. The geometric means of the stimulus values were $6.77,11.25$, and 8.68 for Ranges $\mathrm{A}, \mathrm{B}$, and $\mathrm{C}$ at the lower end, and $90.33,75.34$, and 50.50 for Ranges $X, Y$, and $Z$ at the upper end. A geometric mean hypothesis would predict larger exponents at the higher location, which is the opposite of our results.

To further examine the role of location, the effect of changes in stimulus range should be evaluated for similar locations. For example, the same range can be constructed either by adding small stimuli to a common core of stimuli or by adding large stimuli to that core. Because of the location effect, the exponent should be higher when the range is increased by adding smaller stimuli than when the range is increased by adding larger stimuli. In the present study, as in others (e.g., Algom \& Marks, 1990), range was not manipulated in an orderly fashion. Here, range was increased by adding either smaller stimuli, larger stimuli, or both, thereby preventing a separate evaluation of the effect of each.

Although there was a location effect, and in some cases a range effect, the two appear to be independent of each other. The range effect occurred in the free-modulus condition only, whereas the location effect occurred in all the modulus conditions and was of similar magnitude in all three. One should be aware that increasing (decreasing) the stimulus range by extending (constricting) it downward may have different consequences than extending (constricting) it upward.

Variations in response magnitude do not appear to be responsible for the range effect. Neither the exponent nor the range effect in the free-modulus condition was influenced by the magnitude of a subject's responses. And when a modulus was used to inflate subjects' responses, 
the magnitude of their responses did in fact increase, but the range effect was eliminated.

A constant response range hypothesis also cannot explain the range effect. According to this hypothesis, there should have been no increase in response range in the freemodulus condition. However, log response range increased in all modulus conditions and was similar, in most cases, across modulus conditions.

The fact that the modulus eliminated the range effect has some importance for psychophysics. Whether it was the use of a modulus, the value of the modulus, or the continuous presence of the standard throughout the experimental sessions, the range effect was controlled. However, the location effect occurred both with and without a modulus, implying that this effect may be the more robust one. Should one want psychophysical functions that are not contaminated by stimulus range, one should consider the use of a modulus. We should note that these results were obtained for only one stimulus continuum, two values of modulus, and for ranges and locations that overlapped. Further examination of these factors and their effect on judgments of stimulus intensity shuuld extend to other continua, moduli, and locations.

\section{REFERENCES}

Ahlström, R., \& Baird, J. C. (1989). Shift in stimulus range and the exponent of the function for loudness. Perception \& Psychophysics, 46, 603-607.

Algom, D., \& MaRks, L. E. (1990). Range and regression, loudness scales, and loudness processing: Toward a context-bound psychophysics. Journal of Experimental Psychology: Human Perception \& Performance, 16, 706-727.

Buorkman, M., \& Strangert, B. (1960). The relationship between ratio estimates and stimulus dispersion. Reports from the Psychological Laboratory, 81, 1-8. University of Stockholm.

ENGEN, T. (1956). An evaluation of a method for developing ratio scales. American Journal of Psychology, 69, 92-95.

ENGEN, T., \& LEVy, N. (1958). The influence of context on constantsum loudness judgments. American Journal of Psychology, 71, $731-736$.

EnGEN, T., \& Ross, B. M. (1966). Effect of reference number on magnitude estimation. Perception \& Psychophysics, 1, 74-76.
Fagot, R. F., \& Pokorny, R. (1989). Bias effects on magnitude and ratio estimation power function exponents. Perception \& Psychophysics, 45, 221-230.

Foley, H. J., Cross, D. V., Foley, M. A., \& Reeder, R. (1983). Stimulus range, number of categories, and the "virtual" exponent. Perception \& Psychophysics, 34, 505-512.

Foley, H. J., Cross, D. V., \& O'Reilly, J. A. (1990). Pervasiveness and magnitude of context effects: Evidence for the relativity of absolute magnitude estimation. Perception \& Psychophysics, 48, 551-558.

Gescheider, G. A. (1985). Psychophysics: Method, theory, and application (2nd ed.). Hillsdale, NJ: Erlbaum

JoNES, F. N., \& Woskow, M. J. (1966). Some effects of context on the slope in magnitude estimation. Journal of Experimental Psychology, 71, 170-176.

KunNaPas, T. (1960). Scales for subjective distance. Scandinavian Journal of Psychology, 1, 187-192.

MacMillan, N. A., Moschetto, C. F., Bialostozky, F. M., \& ENGEL, L. (1974). Size judgment: The presence of a standard increases the exponent of the power law. Perception \& Psychophysics, 16, 340-346.

Marks, L. E. (1988). Magnitude estimation and sensory matching. Perception \& Psychophysics, 43, 511-525.

Poulton, E. C. (1968). The new psychophysics: Six models for mag nitude estimation. Psychological Bulletin, 69, 1-19.

Poulton, E. C. (1989). Bias in quantifying judgments. Hillsdale, NJ: Erlbaum.

Stevens, J. C., \& Tulving, E. (1957). Estimations of loudness by a group of untrained observers. American Journal of Psychology, 70, 600-605.

Stevens, S. S. (1971). Issues in psychophysical measurement. Psychological Review, 78, 426-450.

Stevens, S. S., \& Galanter, E. H. (1957). Ratio scales and category scales for a dozen perceptual continua. Journal of Experimental Psychology, 54, 377-411.

TEGHTSOONIAN, R. (1973). Range effects in psychophysical scaling and a revision of Stevens' law. American Journal of Psychology, 86, 3-27.

Teghtsoonian, R., \& Teghtsoonian, M. (1978). Range and regression effects in magnitude scaling. Perception \& Psychophysics, 24, 305-314.

WIEST, W. M., \& BELL, B. (1985). Stevens' exponent for psychophysical scaling of perceived, remembered, and inferred distance. Psychological Bulletin, 98, 457-470.

WoNG, R. (1963). Effect of the modulus on estimates of magnitude of linear extent. American Journal of Psychology, 76, 511-512.

(Manuscript received May 11, 1992; revision accepted for publication March 2, 1993.) 